\title{
THE EXOPLANET REVOLUTION
}

n Yamila Miguel - Leiden Observatory, Leiden, The Netherlands - DOI: https://doi.org/10.1051/epn/2019506

Hot Jupiters, super-Earths, lava-worlds and the search for life beyond our solar system: the exoplanet revolution started almost $\mathbf{3 0}$ years ago and is now taking off.

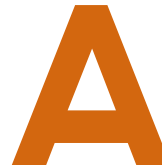

re there other planets like the Earth out there? This is probably one of the oldest questions of humanity. For centuries and until the 90s, we only knew of the existence of 8 planets. But today we live in a privileged time. For the first time in history we know that there are other planets orbiting distant stars.

The first planet orbiting a star similar to the Sun was discovered in 1995 -only 24 years ago- and it started a revolution in Astronomy. Today astronomers have discovered the astonishing number of 4000 exoplanets, and counting. Every new discovery shows an amazing diversity that impacts in the perception and understanding of our own solar system.

\section{How to find exoplanets?}

Finding exoplanets is an extremely difficult task. These planets shine mostly due to the reflection of the stellar light in their atmospheres and their light is incredibly weak compared to that of their host stars. For this reason,
Artist's impression of COROT-7b.

(c) ESO/L. Calçada 
v FIG. 1: Schematic view of the transit

(top panel) and radial velocities methods

(bottom panel). observing exoplanets directly is extremely difficult and astronomers had to develop indirect techniques that infer the presence of the planet.

Two of the most successful techniques to discover exoplanets are the "Transits" and "Radial Velocities" techniques.

In the first one, astronomers observe the dimming of stellar light when the planet passes in front of it (figure 1, top panel). Current instrumentation allows astronomers to measure less than $1 \%$ change in stellar light. Because the portion of stellar light that diminishes is proportional to the size of the planet, this technique allows astronomers to know the planetary radius.

In the Radial Velocities technique, astronomers measure the movement of the star -the doppler shift in the stellar light- caused by the presence of a planet orbiting around it (figure 1, bottom panel). With present techniques, astronomers can measure movements of the star of less than $10 \mathrm{~cm} / \mathrm{s}$ (as a reference we usually walk at $1 \mathrm{~m} / \mathrm{s}$ ) [1]. The effect on the star is larger if the planet has a high mass and is located close to the star and is smaller for small planets. Therefore, the radial velocities technique allows us to have an estimation of the mass of the planet. Other methods include direct imaging (looking at the light from the planet directly) and micro-lensing (observing gravitational lensing due to a planet).

Each one of these methods give valuable information to start understanding the variety of planets out there, in particular the combination of both techniques
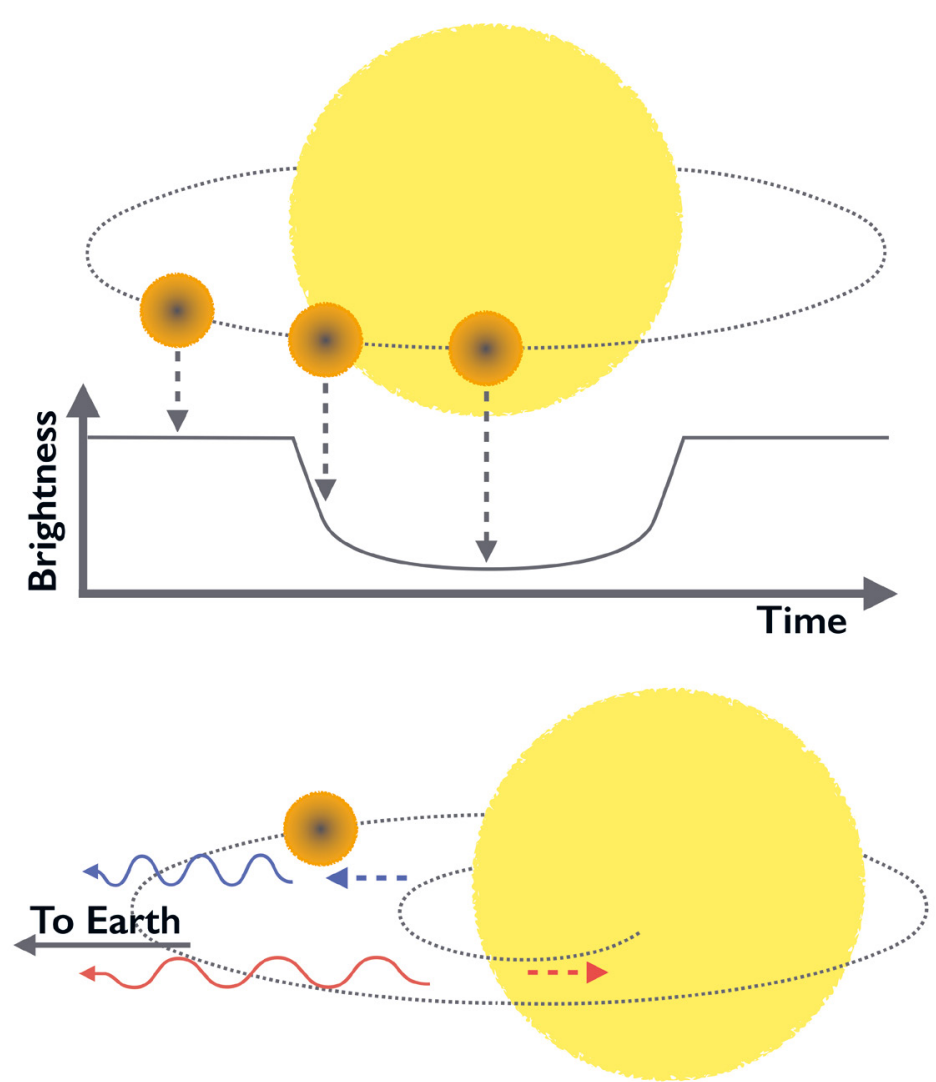

allows astronomers to calculate the planet's density, important to start assessing planetary compositions and diversity.

\section{The Exoplanet Zoo}

The population of exoplanets shows a huge diversity (figure 2). Due to observational biases, most of the planets detected so far orbit very close to their stars -in a few hours- and for this reason many of them are tidally-locked, showing always the same face towards the star, similar to the Moon-Earth system. This affects the circulation of their atmospheres and in some cases creates huge temperature differences between the dayside and dark side that can be as extreme as $\sim 600 \mathrm{~K}$.

The search for exoplanets has revealed other surprises; here we describe some of the strange and unexpected worlds found.

\section{Hot Jupiters and far-away giant planets.}

Astronomers are finding giant planets -like Jupiter and Saturn- but located very close to their stars, much closer than Mercury to the Sun. One of the most famous examples of a "hot-Jupiter" is 51 Pegasus b, the first exoplanet detected around a Sun-type star [2], that orbits its star at a distance of 0.05 times the distance between the Earth and the Sun (Astronomical Unit), which implies that a "year" in that planet lasts 4.2 days.

Astronomers are also finding giant planets very far away from their stars, at approximately twice the distance between Neptune and the Sun, and even further out.

Both hot-Jupiters and far-away giants shook the foundation of planet formation theories, pushing the boundaries and showing that extreme scenarios are possible for the formation and evolution of planets. Today we know that planets are not located in the place where they were born, but that they "migrated" due to the interaction with the protoplanetary disk during their formation and evolution [3]. In addition, some of them might have been scattered away due to the dynamical interaction with the star and other bodies of the planetary system.

\section{Mini-gas planets and super-Earths.}

There are some planets that don't have enough mass to be a giant planet, but are more massive than small rocky planets like our own. These planets have masses of approximately 10 times that of the Earth and for this reason are usually called "Mini-gas planets"-those with a substantial atmosphere made of hydrogen and heliumor "super-Earths" -the ones that are small, and have a much smaller, potentially secondary atmosphere. Since there is no parallel to these planets in our solar system, astronomers don't know what to expect for their interiors, atmospheres or formation history. In addition, analysis of the exoplanet population shows that most of the 
exoplanets discovered so far belong to this category [4], and a lot of effort is going into trying to understand their nature and to know why there are no such planets in our solar System.

\section{Hot rocky exoplanets or "lava worlds".}

These are an intriguing class of planets that are rocky -like the Earth-, but located extremely close to their stars and that might have a magma ocean running on their surfaces. This is caused by the high temperatures on the dayside (of approximately $2000 \mathrm{~K}$ or more) caused by stellar irradiation. In these planets, the magma ocean vaporises and forms an outgassed atmosphere mainly made of vaporised rocks [5] that is escaping the planet in what looks like a cometary tale.

Many of these planets were discovered using powerful ground-based observatories, or space missions like Corot (ESA), Kepler (NASA) or the currently on space TESS mission (NASA). Other space missions such as the upcoming Cheops (ESA, to be launched this year) and JWST (NASA, to be launched in 2021) will help to improve our knowledge on these worlds.

\section{The search for life in the Universe and future prospects}

The ultimate goal of the exploration and search for exoplanets is to know if there are other solar systems and planets like our own. We still don't know how life originated on Earth, but we do know that water is essential for life in our planet. The fact that our planet has oceans of liquid water is due to a combination of different factors, some of them being the mass of our planet, the pressure of our atmosphere and the temperature of the planet, which is a consequence of the gases present in our atmosphere and the irradiation received from the Sun. With this idea in mind, astronomers developed the concept of "habitable zone" [6]. This is the region where a planet with an atmosphere like the Earth should be orbiting its host star in order to maintain liquid water on its surface. Since different stars emit different energy, each star has its habitable zone located at different distances. Keep in mind that if we find an exoplanet with a mass and radius similar to the Earth and located in the habitable zone this doesn't necessarily mean that the planet will host life, it is just saying whether such planet has any possibility of being habitable -having liquid water on its surface- at all. Since different rocky planets might develop different atmospheres, this is just a useful concept to guide our searches, but nothing more. Our current technology allows us to measure only masses and radius of Earthlike exoplanets (and detect some chemical species in bigger, giant exoplanets atmospheres, [7]), therefore it is not yet possible to detect Earth twins and uniquely identify life-forms in exoplanets.

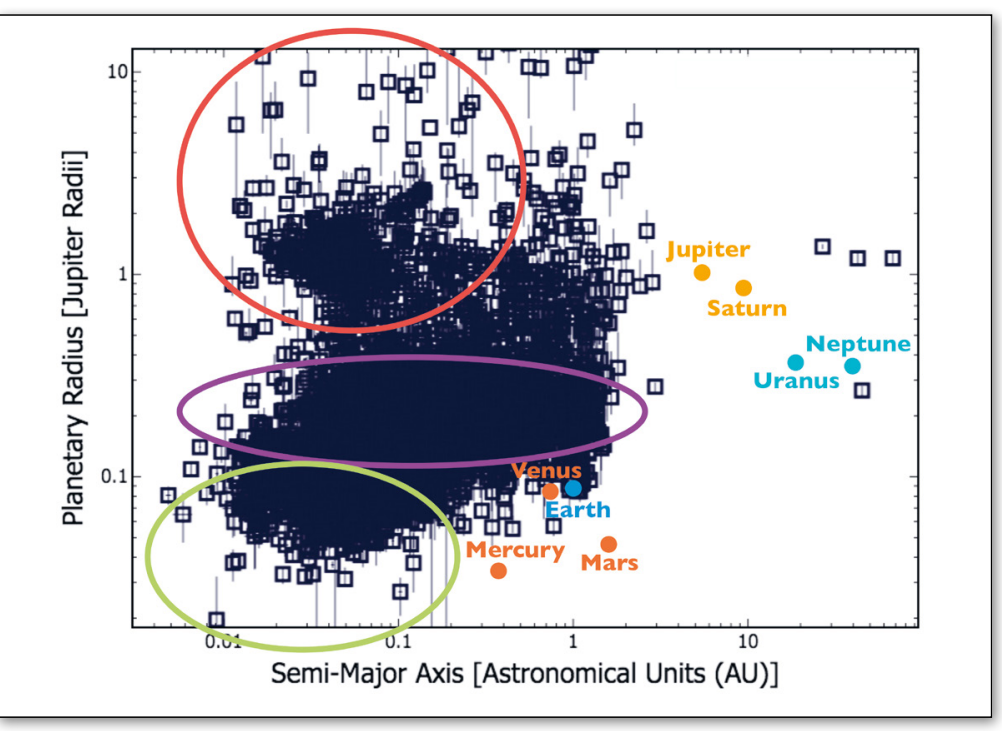

Nevertheless, not everything looks dark in the future, and astronomers are working towards the next generation of instruments that will make this possible [8]. An example is the Plato mission (ESA), a space telescope (to be launched in 2026) that has the goal of finding and characterising planets like the Earth. Examples from the ground, include the next generation of extremely large telescopes (Extremely Large Telescope, Giant Magellan Telescope, Thirty Meter Telescope), that will have mirrors of 30 meters and are currently under construction. These telescopes and the future space missions will lead astronomers to the next step towards finding habitable worlds, where Sci-Fi meets reality.

$\triangle$ FIG. 2: Exoplanets discovered by August 2019 (data from exoplanets.org). The planets in our solar system are indicated with fill circles for comparison. Main population of exoplanets are indicated with circles: hot Jupiters (red), mini-gas giants or super-Earths (purple) and hot rocky. exoplanets (green).

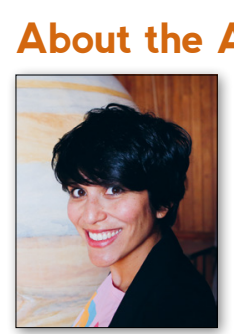

fellow at the Observatoire de la Cote d'Azur (France).

\section{References}

[1] J. I. González Hernández, F. Pepe, P. Molaro and N. C. Santos, Handbook of Exoplanets, 157 (2018)

[2] M. Mayor and D. Queloz, Nature 378, 355 (1995)

[3] D.N.C. Lin and J. Papaloizou, in D.C. Black \& M.S. Matthews, eds., Protostars and Planets // Univ. of Arizona Press, Tucson, pp. 981-1072 (1985)

[4] A. W. Howard, Science 340, 572 (2013)

[5] Y. Miguel, L. Kaltenegger, B. Fegley et al. , ApJL 742, L19 (2011)

[6] J. F. Kasting, D. P. Whitmire and R. T. Reynolds, Icarus 101, 108 (1993)

[7] D. Charbonneau, T. M. Brown, R. W. Noyes et al. , ApJ 568, 377 (2002)

[8] I. A. G. Snellen, R. J. de Kok, R. le Poole et al. , ApJ 764, 182 (2013) 